\title{
FDG-PET/CT pitfalls in gynecological and genitourinary oncological imaging
}

\author{
A Lakhani', S Khan, N Bharwani, V Stewart, A Rockall, T Barwick, S Khan \\ From International Cancer Imaging Society Meeting and 15th Annual Teaching Course (ICIS 2015) \\ London, UK. 5-7 October 2015
}

\section{Learning objectives}

1. To understand the role of FDG PET/CT imaging in the multimodality investigation of gynecological and genitourinary cancers.

2. To describe the mechanism of action and technical pitfalls of FDG-PET/CT.

3. To highlight key imaging features of physiological and non-physiological FDG uptake and show how this is essential for interpretation of gynecological and genitourinary FDG-PET/CT studies.

4. to review the pathophysiological mechanisms leading to potentially false-positive and false-negative assessments.

\section{Content organisation}

Introduction of FDG-PET/CT

- Mechanism of action

- Role in gynecological and genitourinary oncological imaging

- FDG-PET/CT imaging protocols

False positives in gynecological and genitourinary oncological imaging:

- Physiological FDG-PET uptake - pictorial examples of uptake in endometrium and ovaries

- Non-physiological FDG-PET uptake - pictorial examples of pelvic inflammatory disease, fibroids, endometriosis

False negatives in gynecological and genitourinary oncological imaging:

- Physiological FDG-PET uptake - pictorial examples of urinary excretion masking malignant lesions

- No/low FDG uptake - pictorial examples of necrotic lymphadenopathy and low grade tumours

- Artefacts

\footnotetext{
* Correspondence: amishlakhani@gmail.com Imperial College Healthcare NHS Trust, London, UK
}

Pearls explaining how to minimise false interpretation

\section{Conclusion}

FDG-PET/CT has a useful role in gynecological and genitourinary oncological imaging. However, understanding of physiological and non-physiological FDGPET uptake is vital to understand potential false positive and false negatives in interpretation.

FDG PET/CT should be used as one part of the multimodality investigation of gynecological and genitourinary cancers.

Published: 2 October 2015

doi:10.1186/1470-7330-15-S1-P42

Cite this article as: Lakhani et al:: FDG-PET/CT pitfalls in gynecological and genitourinary oncological imaging. Cancer Imaging 2015 15(Suppl 1): P42.
Submit your next manuscript to BioMed Central and take full advantage of:

- Convenient online submission

- Thorough peer review

- No space constraints or color figure charges

- Immediate publication on acceptance

- Inclusion in PubMed, CAS, Scopus and Google Scholar

- Research which is freely available for redistribution
() Biomed Central 